\title{
Well-being and mental health should be top priority for the emergency medicine workforce
}

\author{
Kerstin de Wit ${ }^{1,2}$ (1) $\cdot$ Rodrick Lim $^{3}$
}

Received: 30 May 2021 / Accepted: 5 June 2021

(C) The Author(s), under exclusive licence to Canadian Association of Emergency Physicians (CAEP)/ Association Canadienne de Médecine d'Urgence (ACMU) 2021

In this issue, Gaucher et al. report the results of an emergency physician survey focusing on experiences, concerns and perspectives during the first wave of the pandemic [1]. This well-developed, bilingual survey reminds us of the strain we have lived through over the past 14 months, ranging from concern over a potential second wave to worry about the impact of social isolation on Canada's youth. Respondents had witnessed distress from patients' families, delayed presentations to the emergency department with advanced illness, reduced access to healthcare and patients accessing the emergency department in place of primary care. In contrast to recent experience, physicians had not experienced rationing of intensive care beds. During this first wave, emergency physicians felt a very strong sense of responsibility to care for patients. Despite reporting anxiety, worry, frustration and anger, participants rated their own mental health as low priority.

Emergency physician well-being and burnout has not been extensively studied during this pandemic. A study following Canadian emergency physicians during the first 10 weeks of the pandemic reported high burnout levels in $10-18 \%$ of the participants [2]. Studies from the United States and Singapore reported moderate to high levels of stress and burnout in the early pandemic months $[3,4]$. We lack ongoing data on emergency physician mental health and well-being into the second and now third waves of the pandemic in Canada.

Kerstin de Wit

Kerstin.dewit@queensu.ca

1 Department of Emergency Medicine, Queen's University, Kingston, ON, Canada

2 Department of Medicine and Department of HEI, McMaster University, Hamilton, ON, Canada

3 Departments of Paediatrics and Medicine, University of Western Ontario, London, ON, Canada
The pandemic has had so many waves over such a prolonged time, that it is important to study and understand the specific factors in each stage which deeply affect physician mental health. Provinces undergoing a third wave have been particularly affected as they have faced redeployment, the possibility of critical care triage and the knowledge that the lack of public health measures and compliance may have facilitated COVID-19 spread, all factors that lead to moral distress.

As we ride the third wave of this pandemic, it is clear that neglecting our own mental health is counterproductive for the sustainability and longevity of our work efforts. An early pandemic study of health care workers in Lombardy, Italy showed that $40 \%$ fulfilled a provisional diagnosis of post-traumatic stress disorder (PTSD) [5]. Those categorized as languishing (poor mental health) were three times more likely than those with moderate mental health to be diagnosed with PTSD. This is a huge area of concern to emergency medicine, where even prior to the pandemic, physicians suffered high levels of burnout. A 2019 Canadian emergency physician survey categorized $16 \%$ of participants as moderate or severely depressed [6]. Those who were depressed were much more likely to suffer from burnout.

The long-term health effects for frontline workers postpandemic should be a significant concern to government, hospitals, and authorities. Future health systems and their patients rely on healthy frontline workers to provide timely, effective care. This will be even more important as the COVID-19 pandemic abates, and our hospitals address large volumes of patients who have had their general health neglected during the pandemic.

Hopefully as we begin to see the other side of this pandemic, a new focus on physician health and sustainability will emerge. If you think you are struggling, please consider accessing wellness resources to help you and your loved ones. The Canadian Medical Association has a Wellness Support Line (https://www.cma.ca/supportline) which is open to medical students, residents, practicing physicians 
and family members which can direct you to resources by province/territory. The Canadian Association of Emergency Physicians also provides a COVID-19 Wellness Resources section on their website (https://caep.ca/covid-19-wellnessresources).

\section{Declaration}

Conflict of Interest The authors have no conflicts of interest.

\section{References}

1. Gaucher N, Trottier ED, Côté AJ, et al. A survey of Canadian emergency physicians' experiences and perspectives during the COVID-19 pandemic. Can J Emerg Med. 2021. https://doi.org/ 10.1007/s43678-021-00129-4.
2. de Wit K, Mercuri M, Wallner C, et al. Canadian emergency physician psychological distress and burnout during the first 10 weeks of COVID-19: a mixed-methods study. JACEP Open. 2020;1:1030-8.

3. Rodriguez RM, Medak AJ, Baumann BM, et al. Academic emergency medicine physicians' anxiety levels, stressors, and potential stress mitigation measures during the acceleration phase of the COVID-19 pandemic. Acad Emerg Med. 2020;27(8):701-7.

4. Chor WPD, Ng WM, Cheng L, et al. Burnout amongst emergency healthcare workers during the COVID-19 pandemic: A multicenter study. Am J Emerg Med. 2020. https://doi.org/10.1016/j. ajem.2020.10.040.

5. Bassi M, Negri L, DelleFave A, Accardi R. The relationship between post-traumatic stress and positive mental health symptoms among health workers during COVID-19 pandemic in Lombardy, Italy. J Affect Dis. 2021;280:1-6.

6. Lim R, van Aarsen L, Gray S, Rang L, Fitzpatrick J, Fischer L. Emergency medicine physician burnout and wellness in Canada before COVID19: a national survey. CJEM. 2020;22(5):603-7. 